\title{
An Image Segmentation by BFV and TLBO
}

\author{
Mohammad Heidari \\ Department of Mechanical Engineering, Abadan Branch, Islamic Azad University, Abadan, Iran
}

Correspondence should be addressed to Mohammad Heidari; moh104337@yahoo.com

Received 3 August 2016; Accepted 13 October 2016

Academic Editor: Mehmet Onder Efe

Copyright (C) 2016 Mohammad Heidari. This is an open access article distributed under the Creative Commons Attribution License, which permits unrestricted use, distribution, and reproduction in any medium, provided the original work is properly cited.

\begin{abstract}
This paper presents the establishing of a biconvex fuzzy variational (BFV) method with teaching learning based optimization (TLBO) for geometric image segmentation (GIS). Firstly, a biconvex object function is adopted to process GIS. Then, TLBO is introduced to maximally optimize the length penalty item (LPI), which will be changed under teaching and learner phase of TLBO, making the LPI closer to the target boundary. Afterward, the LPI can be adjusted based on fitness function, namely, the evaluation standards of image quality. Finally, the LP is combined item with the numerical order to get better results. Different GIS strategies are compared with various fitness functions in terms of accuracy. Simulations show that the presented method is more effective in this area.
\end{abstract}

\section{Introduction}

Image segmentation is very important in image processing. It is also an important research direction of computer vision technology and has been highly appreciated by people for many years. On the one hand, it is the foundation of target expression and has an important influence on the measurement of characteristics; on the other hand, because of the image segmentation and expression of segmentation, feature extraction and target parameter measurement based on segmentation will convert the original image into more abstract and more compact form, making it possible for higher level of image analysis and understanding. There are many methods in the field of image segmentation such as thresholding [1], edge detection of image [2], clustering for image [3, 4], regional active contour [5], and specific mathematical theory tools [6]. Some new segmentation methods were also proposed such as anterior cruciate ligament [7], improved firefly algorithm [8], and fuzzy $c$-means clustering for image detection [9]. They are relatively simple image segmentation methods and most widely used, but there are still various deficiencies. The active contour model without edges named $\mathrm{CV}$ model is one of the most successful models in image segmentation. However, CV model also has drawbacks: (1) converging to local optima [10], (2) being sensitive to selection of parameters [11], and (3) computational inefficiency [12]. In order to overcome these drawbacks, a novel BFV image can be proposed [13], but the BFV method is only suitable for some special images and the LPI is randomly initialized; there is no universality. The paper proposes an efficient biconvex fuzzy method with TLBO for image segmentation. The TLBO approaches [14-16] used to maximally optimize the LPI will be changed under teaching phase and learner phase to get better results. Rao $[17,18]$ presented the Jaya algorithm in this area for optimization of the problem.

The article is organized as follows. Section 2 describes the CV model and the BFV model. Section 3 describes the TLBO strategy. Section 4 presents GIS algorithm based on BFV by TLBO. In Section 5, experimental results of the proposed model are given. Section 6 depicts the conclusion of this paper.

\section{CV Model and the BFV Model}

Chan and Vese [11] simplified the Mumford-Shah model, and they presented a novel active contour model based on region, namely, the CV model [19-21]. The model assumes that the image is divided into two types of target and background. The energy function is defined as follows:

$$
\begin{aligned}
& E(C)=\mu L(C)+\nu A(C)+\lambda_{1} \int_{i(C)}\left|I(x, y)-C_{1}\right|^{2} d x \\
& +\lambda_{2} \int_{o(C)}\left|I(x, y)-C_{2}\right|^{2} d y,
\end{aligned}
$$


where $E, I(x, y)$, and $C$ are the energy of image, original image, and contour of the original image, respectively. $i(C)$ and $o(C)$ show the region, which are inside and outside the contour. The latter two items are fitted to detect the target region and background region. $L(C)$ indicates the length of the contour. $A(C)$ shows the area inside the contour. $C_{1}$ indicates the average gray inside the contour and $C_{2}$ shows the average gray outside the contour. Also, $\mu, v, \lambda_{1}$, and $\lambda_{2}$ are all nonnegative numbers, where $\lambda_{1}$ and $\lambda_{2}$ are weights for the LPI and the fitting item, respectively. To minimize the energy function and achieve the best effect, fuzzy energy functional is used as follows:

$$
\begin{aligned}
E & =\lambda_{1} \int_{\Omega} \mu^{m}\left(I-C_{1}\right)^{2} d x \\
& +\lambda_{2} \int_{\Omega}(1-\mu)^{m}\left(I-C_{2}\right)^{2} d y .
\end{aligned}
$$

In formula (2), $\mu$ and $m$ are the membership function and a constant positive integer, respectively. In this paper, $\lambda_{1}=$ $\lambda_{2}=1$. The first-order partial derivatives in formula (2) are calculated with respect to $C_{1}$ and $C_{2}$, and then they are set to be zero. $C_{1}$ and $C_{2}$ are as follows:

$$
\begin{aligned}
& C_{1}=\frac{\int_{\Omega} \mu^{m} I d x}{\int_{\Omega} \mu^{m} d x}, \\
& C_{2}=\frac{\int_{\Omega}(1-\mu)^{m} I d x}{\int_{\Omega}(1-\mu)^{m} d x} .
\end{aligned}
$$

Another shortcoming of the CV model is that the LPI restricts the choice of the initial value for level set function. Also, the selection of initial level set function depends on $\mu$, so the proposed method is using TLBO to optimize the length item. Calculating the Gateaux derivative to energy functional according to formula (1) and derivative function on variables $\delta$, the level set function is expressed as $\phi$, which shows the initial surface of the contour. It should be noted that the zero level set is the contour. The evolution process is to get the derivative of the function $\phi$ on variable $t$, as shown in the following:

$$
\begin{aligned}
\frac{\partial \phi}{\partial t} & =\delta(\phi)\left[\mu \operatorname{div}\left(\frac{\nabla \phi}{|\nabla \phi|}\right)-v-\lambda_{1}\left(1-C_{1}\right)^{2}\right. \\
& \left.+\lambda_{2}\left(1-C_{2}\right)^{2}\right] .
\end{aligned}
$$

In formula (4), $\operatorname{div}, \delta(\phi)$, and $\nabla$ are divergence operator, approximating solution of Dirac function, and gradient operator, respectively.

In the experiment, $v=0$ and $\lambda_{1}=\lambda_{2}=1$. In the same way, the evolution function for level set of formula (1) is

$$
\begin{aligned}
& \frac{\partial \mu}{\partial t} \\
& \quad=m\left(-\lambda_{1} \mu^{m-1}\left(I-C_{1}\right)^{2}+\lambda_{2}(1-\mu)^{m-1}\left(I-C_{2}\right)^{2}\right) .
\end{aligned}
$$

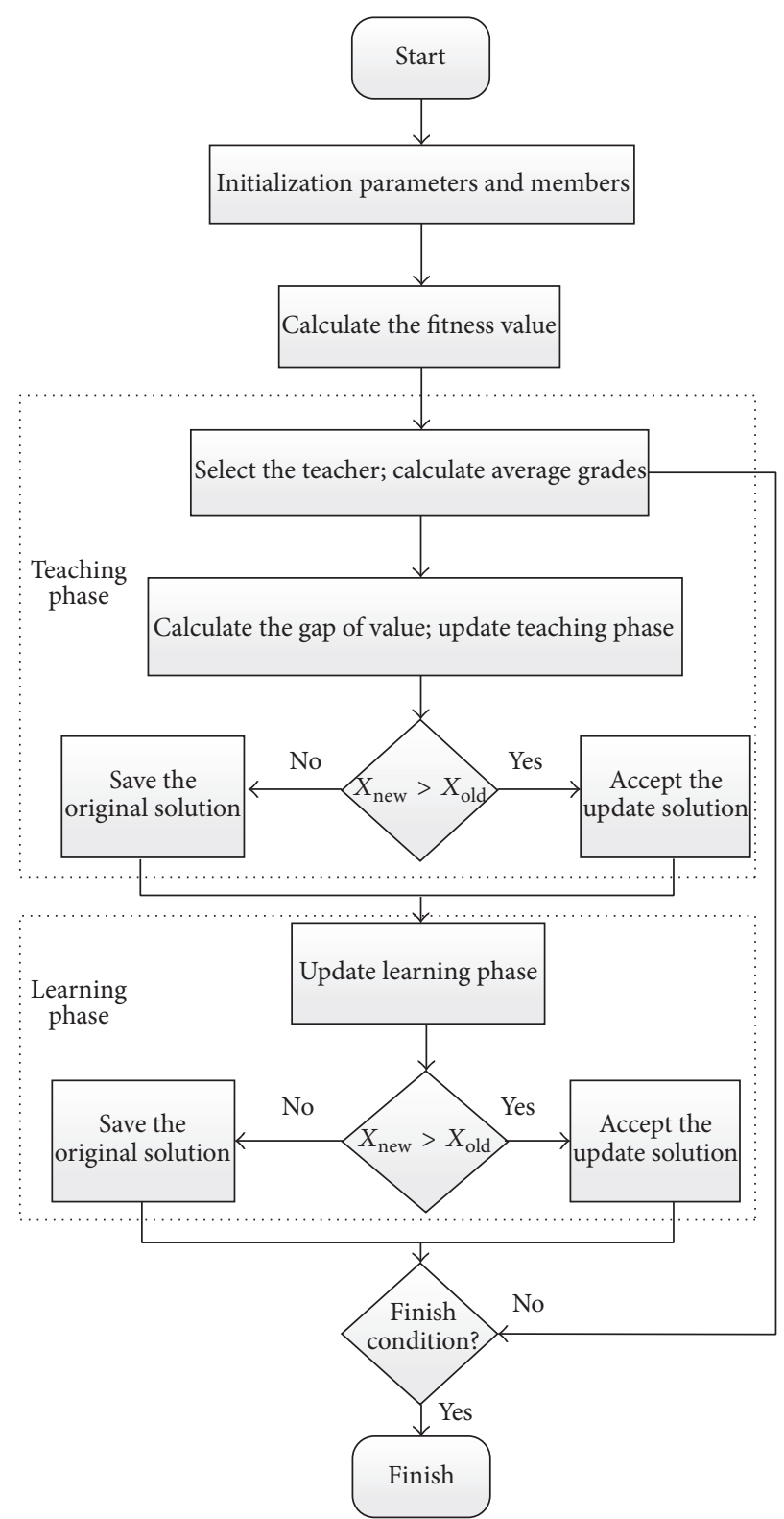

FIgURE 1: Algorithm of TLBO.

In this paper, standard Von Neumann analysis is applied for studying the time stability [22]. The following formula is used for calculation:

$$
\begin{aligned}
\mu_{n} & =\mu_{n+1}+\Delta \operatorname{tg}(p(|\nabla I, s|), s) \Delta \mu_{n+1 / 2}, \\
p(|\nabla I, s|) & =\varepsilon\left(s-s_{0}\right)|\nabla I|+\left(1-\varepsilon\left(s-s_{0}\right)\right)|G * \nabla I|, \\
\varepsilon(s) & =\frac{1}{1+e^{-s}}, \\
g_{\max } & =\max \{g(p(|\nabla I|))\},
\end{aligned}
$$

where $s, G$, and $*$ are the SNR of test image, Gaussian kernel, and operation of convolution, respectively. 


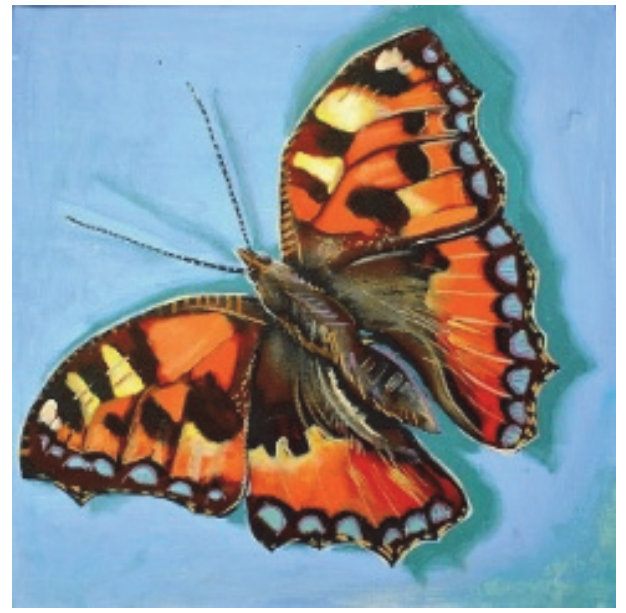

(a)

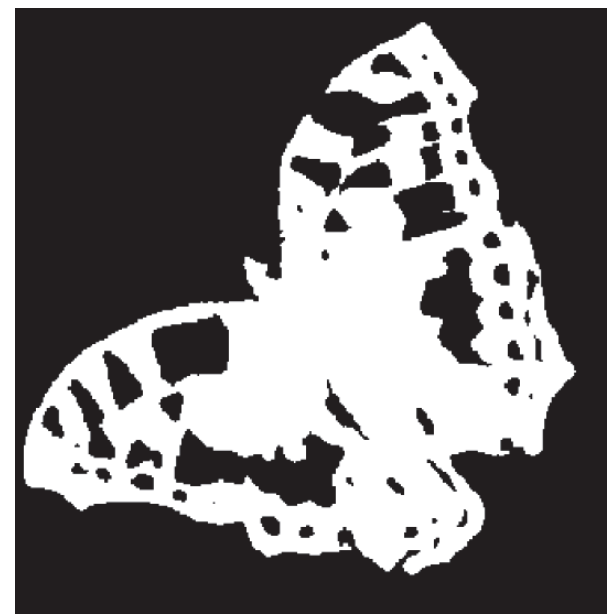

(c)

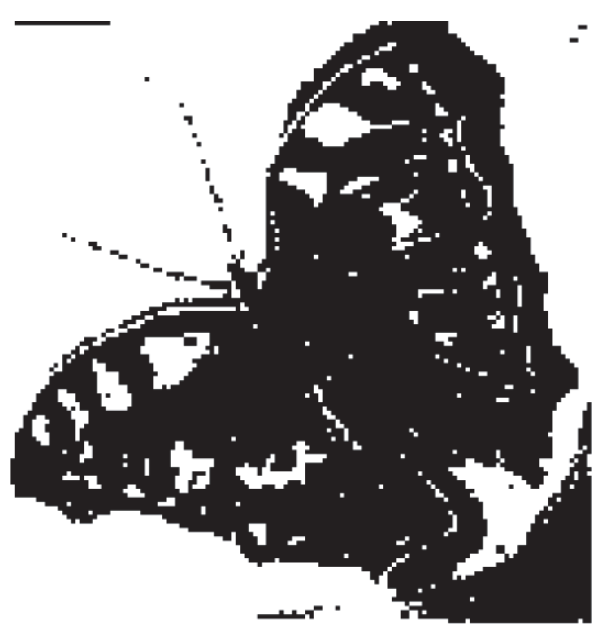

(b)

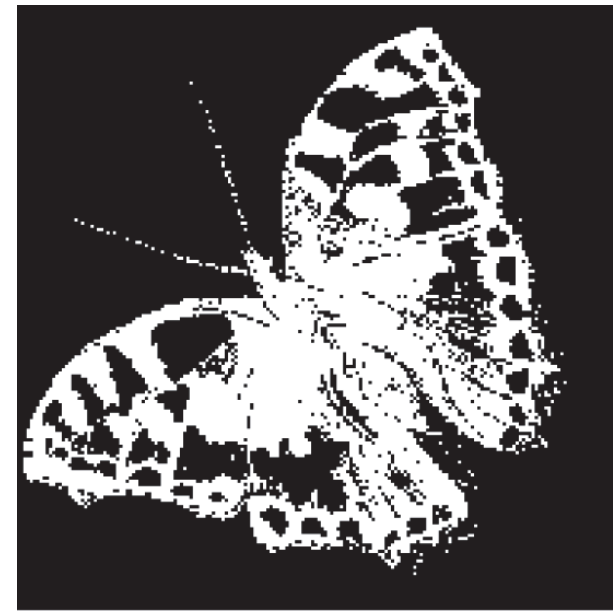

(d)

Figure 2: (a) Primary image. (b) CV segmentation. (c) BFV segmentation. (d) TLBO-BFV segmentation.

\section{TLBO}

The TLBO put forward by Rao et al. [14-16] is a novel heuristic algorithm. The model can be described that it randomly generated a series of solutions in the constraints space. These solutions can be regarded as a group of "students," and one of the best is recognized as a "teacher." The teacher imparts knowledge and answers students' questions. Students enrich their knowledge from the teacher. This is the first process of TLBO algorithm [19], called the teaching phase. The learning phase, which is the second process, can be described as communicating with others and exchanging experience to promote each other. After a period of time, the students' knowledge is higher and higher; that is to say, it is more and more tending to the optimal solution in the constraint space. The whole process is shown in Figure 1. The optimal model is as follows:
$g_{i}(X) \leq 0$,

$(i=1,2, \ldots, m)$,

where $f(X)$ is optimized objective function, searching any point $X^{j}=\left(x_{1}, x_{2}, \ldots, x_{d}\right), j=1,2, \ldots, S_{p}, S_{p}$ is the number of species, and $d$ are dimensions of $X$. Continuous variables $S=\left\{X \mid x_{q}^{L} \leq x_{q} \leq x_{q}^{U}, q=1,2, \ldots, d\right\}$, and $x_{q}^{L}$ and $x_{q}^{U}$ are lower and upper bound of each dimension weight of $X$, respectively. Discrete variable $x_{q} \in S=\left\{X_{1}, X_{2}, \ldots, X_{p}\right\}$, and $p$ is a number of discrete set. In the TLBO algorithm, the relations of class, students, and teacher are as follows:

Class: set $\left\{X^{j}, j=1,2, \ldots, S_{p}\right\}$.

Students: set $X^{j}=\left(x_{1}, x_{2}, \ldots, x_{d}\right)$, where $d$ is the subject of teaching.

$$
\min f(X) \text { or } \max f(X)
$$

Subject to $X \in S$
Teacher: the best fitness value $f(X)$ of set $\left\{X^{j}, j=\right.$ $\left.1,2, \ldots, S_{p}\right\}$. 


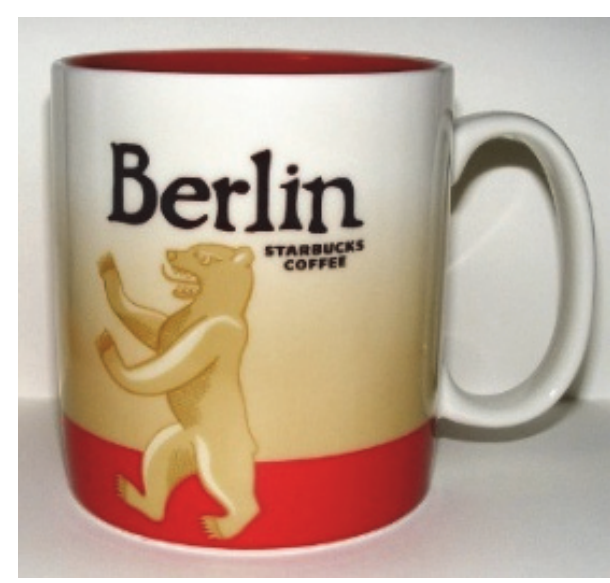

(a)

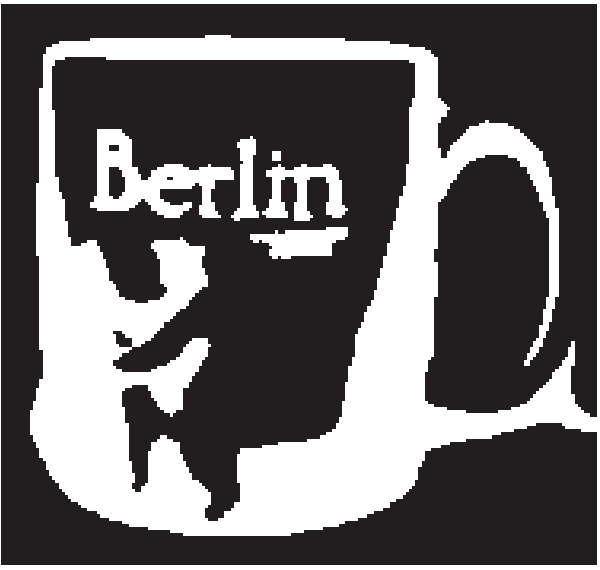

(c)

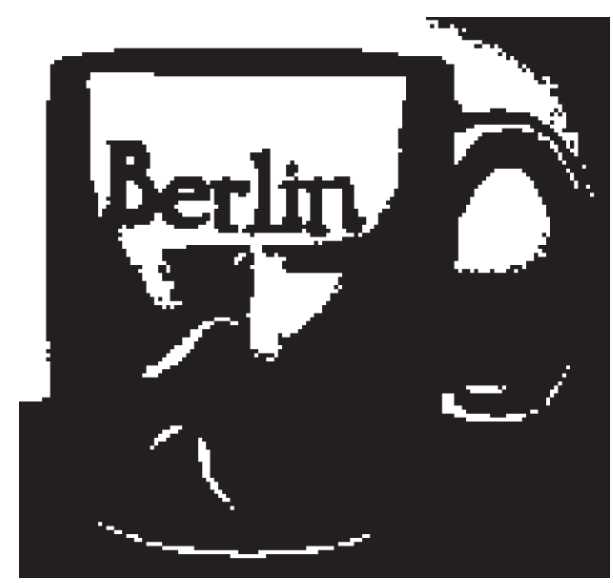

(b)

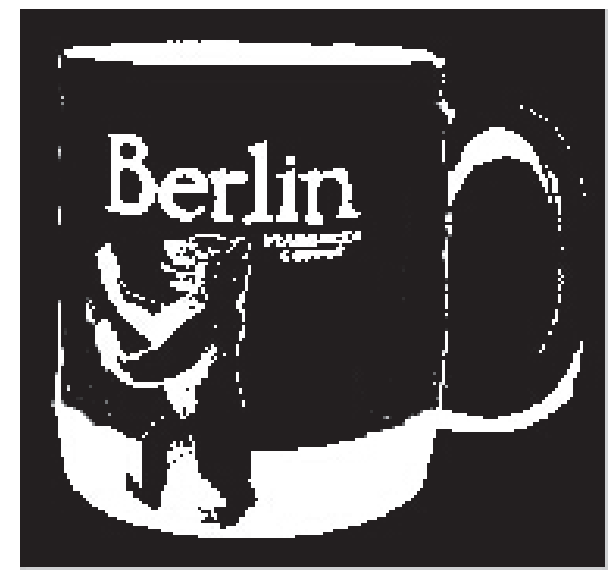

(d)

Figure 3: (a) Primary image. (b) CV segmentation. (c) BFV segmentation. (d) TLBO-BFV segmentation.

The class matrix is defined as follows:

$$
\begin{gathered}
\left(\begin{array}{c|c}
f\left(X^{1}\right) & X^{1} \\
f\left(X^{2}\right) & X^{2} \\
\vdots & \vdots \\
f\left(X^{S_{p}}\right) & X^{S_{p}}
\end{array}\right)=\left(\begin{array}{cccc}
x_{1}^{1} & x_{2}^{1} & \cdots & x_{d}^{1} \\
x_{1}^{2} & x_{2}^{2} & \cdots & x_{d}^{2} \\
\vdots & \vdots & \cdots & \vdots \\
x_{1}^{S_{p}} & x_{2}^{S_{p}} & \cdots & x_{d}^{S_{p}}
\end{array}\right) \\
X_{\text {teacher }}=\min f\left(X^{j}\right), \\
\quad j=1,2, \ldots, S_{p} .
\end{gathered}
$$

The marks distribution curve of the class is a normal distribution, as in formulation (6); in spite of having certain deviation with the actual, it still be helpful for analysis. The definition above can be described as

$$
f(X)=\frac{1}{\sigma \sqrt{2 \pi}} e^{-(x-\mu)^{2} / 2 \sigma^{2}} .
$$

In formula (9), $\sigma, x$, and $\mu$ are the variance, the value in certain range, and the mean value, respectively.
3.1. Teaching and Learner Phase. In the beginning of teaching phase, students' values are relatively scattered and the average grades are $S_{A}$; at the same time, the teacher' is $T_{A}$. After a period of teaching, students' results gradually concentrate distribution, and the average grade is increasing to $S_{B}$, and the teacher is also updated to $T_{B}$. Students learn knowledge from the teacher through the difference between the average of teacher and the student in the teaching phase. In short, the solution is updated with formula (9). If the new solution is better than the existing one, replace the existing solution with the new one.

$$
\text { Difference_Mean }_{\text {new }, i}=r_{i}\left(M_{\text {new }, i}-T_{F} M_{i}\right) \text {, }
$$

where $r_{i}$ is a random number, $r_{i} \in[0,1]$, and $T_{F}$ is a teaching factor which decides how the mean value is to be changed. Because $T_{F}$ is either 1 or 2 , it can be presented as follows:

$$
T_{F}=\operatorname{round}[1+\operatorname{rand}(0,2)]
$$

Solutions of the problem are updated based on the following strategy. At first, the value of objective function is obtained. If the new solution is better than the existing one, replace it 


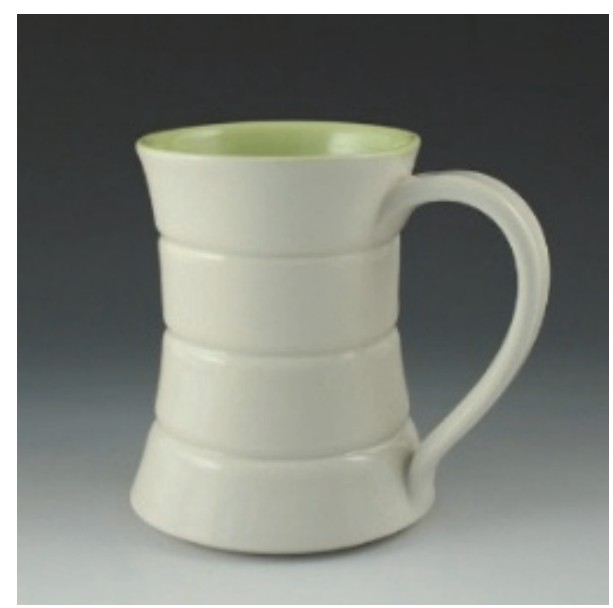

(a)

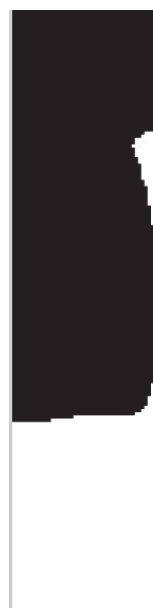

(c)

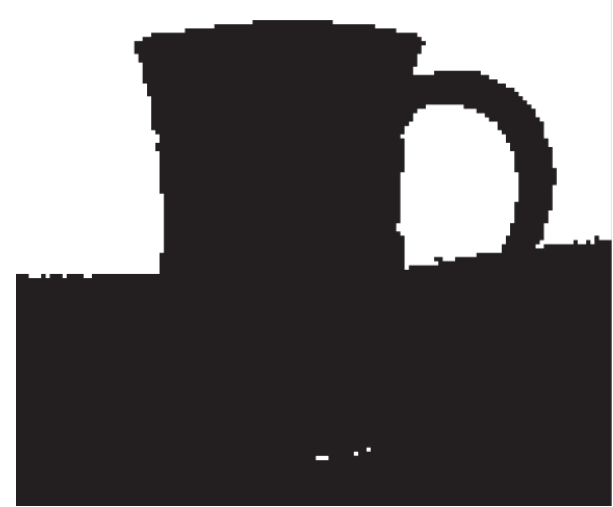

(b)

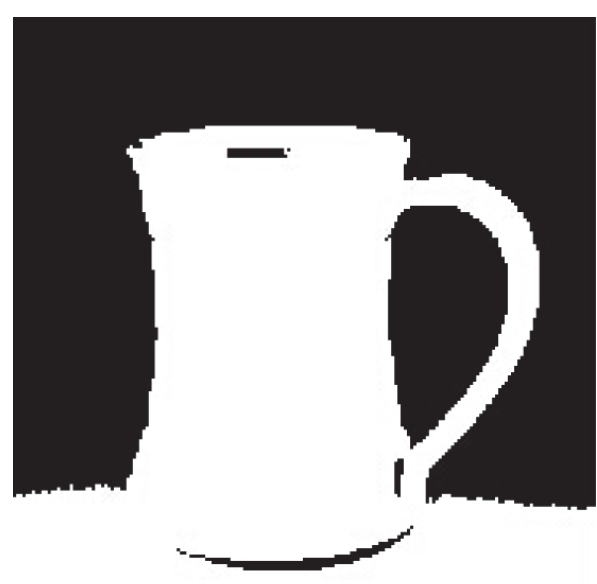

(d)

FIgURE 4: (a) Primary image. (b) CV segmentation. (c) BFV segmentation. (d) TLBO-BFV segmentation.

with the new one:

$$
\begin{aligned}
& \text { ii random (pop_rize) }(i i \neq i) \\
& \text { if } X_{i} \text { is better than } X_{i i} \text { then } \\
& X_{i, \text { new }}=X_{i}+r \cdot\left(X_{i}-X_{i i}\right) \\
& \text { else } \\
& X_{i \text {,new }}=X_{i}+r \cdot\left(X_{i i}-X_{i}\right) \\
& \text { end if } \\
& \text { evaluate }\left(X_{i, \text { new }}\right) \\
& \text { if } X_{i, \text { new }} \text { is better than } X_{i} \text {, then } \\
& X_{i} \leftarrow X_{i, \text { new }} \\
& \text { end if }
\end{aligned}
$$

Figure 1 show the flowchart of TLBO algorithm.

\section{GIS Strategy Based on BFV with TLBO}

In this paper, the fitness function applies Jaccard Similarity (JS) [23] value and standard deviation (Std) as the quantitative indexes to evaluate the results. The JS value is explained as

$$
\mathrm{JS}\left(S_{1}, S_{2}\right)=\frac{\left|S_{1} \cap S_{2}\right|}{\left|S_{1} \cup S_{2}\right|} .
$$

In (12), $S_{2}$ and $S_{1}$ are the ground truth data and the segmentation results, respectively. The Std is defined as

$$
\operatorname{Std}=100 \times \frac{1}{\left((1 /(n-1)) \sum_{i=1}^{n}\left(x_{i}-\bar{x}\right)^{2}\right)^{1 / 2}}
$$

where $\bar{x}$ and $x_{i}$ are the mean of image pixels and the value of image pixels, respectively. From the discussion above, it is not necessary to record experimental results each time. We can take a reaction part to $T$ with step $\Delta t(T=n \Delta t$, where $n$ is a positive integer). In summary, the flowchart of 


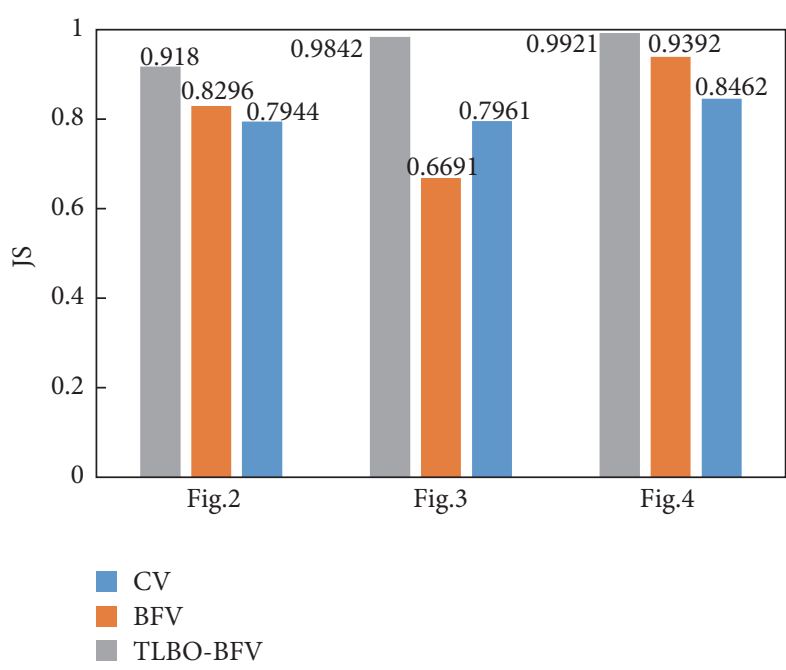

FIGURE 5: JS of methods.

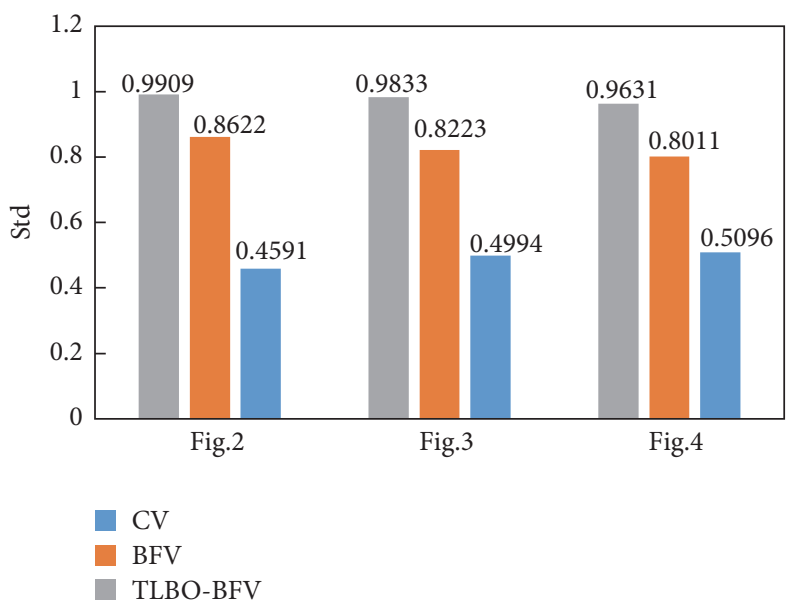

Figure 6: Standard deviation of methods.

the proposed algorithm (TLBO-BFV) can be summed up as in the following steps:

(1) Set $n=0$. It uses the TLBO method to optimize $u$ and calculate $g$ using formula (6).

(2) Calculate $C_{1}$ and $C_{2}$ using formula (3).

(3) Set $t=0$. Compute $\mu^{n+1 / 2}$ using formula (5). If $t+\Delta t \leq$ $T$, then set $t=t+\Delta t$ and $\mu_{n}=\mu_{n+1 / 2}$ and it goes to step (2); otherwise, it continues.

(4) It computes $\mu_{n+1}$ using formula (6).

(5) If $\mu_{n+1}$ satisfy termination condition, it stops; otherwise, set $n=n+1$ and it returns to step (2).

\section{Simulation}

The CV model and BFV segmentation method are selected to compare with TLBO-BFV method. In the experiments, the same iteration number is set.

Tables 1, 2, and 3 demonstrate the results of Figures 2, 3, and 4 , respectively.
TABLE 1: Results of Figure 2.

\begin{tabular}{lcc}
\hline Model & Std & JS \\
\hline CV & 0.4591 & 0.7944 \\
BFV & 0.8622 & 0.8296 \\
TLBO-BFV & 0.9909 & 0.918 \\
\hline
\end{tabular}

TABLE 2: Results of Figure 3.

\begin{tabular}{lcc}
\hline Model & Std & JS \\
\hline CV & 0.4944 & 0.7961 \\
BFV & 0.8233 & 0.6691 \\
TLBO-BFV & 0.9833 & 0.9842 \\
\hline
\end{tabular}

TABLE 3: Results of Figure 4.

\begin{tabular}{lcc}
\hline Model & Std & JS \\
\hline CV & 0.5096 & 0.8462 \\
BFV & 0.8011 & 0.9392 \\
TLBO-BFV & 0.9631 & 0.9921 \\
\hline
\end{tabular}

Figures 2, 3, and 4 show the value of JS and Std of the three methods.

Figures 5 and 6 demonstrate the JS and Std of the three methods, respectively.

\section{Conclusion}

This paper presents a new image segmentation method, named TLBO-BFV. The TLBO approach is used to maximally optimize the LPI, so as to improve the accuracy of segmentation. Simulations indicate that the proposed method improves the robustness and the recognition rate and the algorithm has advantages in stability and effectiveness.

\section{Competing Interests}

The author declares that they have no competing interests.

\section{References}

[1] S. Patra, R. Gautam, and A. Singla, "A novel context sensitive multilevel thresholding for image segmentation," Applied Soft Computing Journal, vol. 23, pp. 122-127, 2014.

[2] C. I. Gonzalez, P. Melin, J. R. Castro, O. Castillo, and O. Mendoza, "Optimization of interval type-2 fuzzy systems for image edge detection," Applied Soft Computing Journal, vol. 47, pp. 631-643, 2016.

[3] J. Hou, W. Liu, X. Ex, and H. Cui, "Towards parameterindependent data clustering and image segmentation," Pattern Recognition, vol. 60, pp. 25-36, 2016.

[4] X. Fan, L. Ju, X. Wang, and S. Wang, "A fuzzy edge-weighted centroidal Voronoi tessellation model for image segmentation," Computers \& Mathematics with Applications, vol. 71, no. 11, pp. 2272-2284, 2016.

[5] F. Y. Shih and K. Zhang, "Locating object contours in complex background using improved snakes," Computer Vision and Image Understanding, vol. 105, no. 2, pp. 93-98, 2007. 
[6] O. Hadjerci, A. Hafiane, N. Morette, C. Novales, P. Vieyres, and A. Delbos, "Assistive system based on nerve detection and needle navigation in ultrasound images for regional anesthesia," Expert Systems with Applications, vol. 61, pp. 64-77, 2016.

[7] H. Lee, H. Hong, and J. Kim, "Segmentation of anterior cruciate ligament in knee MR images using graph cuts with patientspecific shape constraints and label refinement," Computers in Biology and Medicine, vol. 55, pp. 1-10, 2014.

[8] B. Krawczyk and P. Filipczuk, "Cytological image analysis with firefly nuclei detection and hybrid one-class classification decomposition," Engineering Applications of Artificial Intelligence, vol. 31, pp. 126-135, 2014.

[9] X. L. Jiang, Q. Wang, B. He, S. J. Chen, and B. L. Li, "Robust level set image segmentation algorithm using local correntropybased fuzzy c-means clustering with spatial constraints," Neurocomputing, vol. 207, pp. 22-35, 2016.

[10] G. Aubert and P. Kornprobst, Mathematical Problems in Image Processing Partial Differential Equations and the Calculus of Variations, Springer, New York, NY, USA, 2006.

[11] T. F. Chan and L. A. Vese, "Active contours without edges," IEEE Transactions on Image Processing, vol. 10, no. 2, pp. 266-277, 2002.

[12] T.-T. Tran, V.-T. Pham, and K.-K. Shyu, "Image segmentation using fuzzy energy-based active contour with shape prior," Journal of Visual Communication and Image Representation, vol. 25, no. 7, pp. 1732-1745, 2014.

[13] M. Gong, D. Tian, L. Su, and L. Jiao, "An efficient bi-convex fuzzy variational image segmentation method," Information Sciences, vol. 293, pp. 351-369, 2015.

[14] R. V. Rao, V. J. Savsani, and D. P. Vakharia, “Teaching-learningbased optimization: a novel method for constrained mechanical design optimization problems," Computer Aided Design, vol. 43, no. 3, pp. 303-315, 2011.

[15] R. V. Rao, V. J. Savsani, and D. P. Vakharia, “Teaching-learningbased optimization: an optimization method for continuous non-linear large scale problems," Information Sciences, vol. 183, no. 1, pp. 1-15, 2012.

[16] R. V. Rao, Teaching Learning Based Optimization and Its Engineering Applications, Springer, Basel, Switzerland, 2016.

[17] R. V. Rao, "Jaya: a simple and new optimization algorithm for solving constrained and unconstrained optimization problems," International Journal of Industrial Engineering Computations, vol. 7, no. 1, pp. 19-34, 2016.

[18] R. V. Rao, K. More, J. Taler, and P. Ocłoń, "Dimensional optimization of a micro-channel heat sink using Jaya algorithm," Applied Thermal Engineering, vol. 103, pp. 572-582, 2016.

[19] R. V. Rao, "Review of applications of tlbo algorithm and a tutorial for beginners to solve the unconstrained and constrained optimization problems," Decision Science Letters, vol. 5, no. 1, pp. 1-30, 2016.

[20] B. Al-Diri, A. Hunter, and D. Steel, "An active contour model for segmenting and measuring retinal vessels," IEEE Transactions on Medical Imaging, vol. 28, no. 9, pp. 1488-1497, 2009.

[21] S. K. Nath, K. Palaniappan, and F. Bunyak, "Cell segmentation using coupled level sets and graph-vertex coloring," Medical Image Computing and Computer-Assisted Inter-Vention, vol. 9, no. 1, pp. 101-108, 2006.

[22] K. Zhang, L. Zhang, H. Song, and D. Zhang, "Reinitializationfree level set evolution via reaction diffusion," IEEE Transactions on Image Processing, vol. 22, no. 1, pp. 258-271, 2013.
[23] S. Jimenez, F. A. Gonzalez, and A. Gelbukh, "Mathematical properties of soft cardinality: enhancing Jaccard, Dice and cosine similarity measures with element-wise distance," Information Sciences, vol. 367-368, pp. 373-389, 2016. 

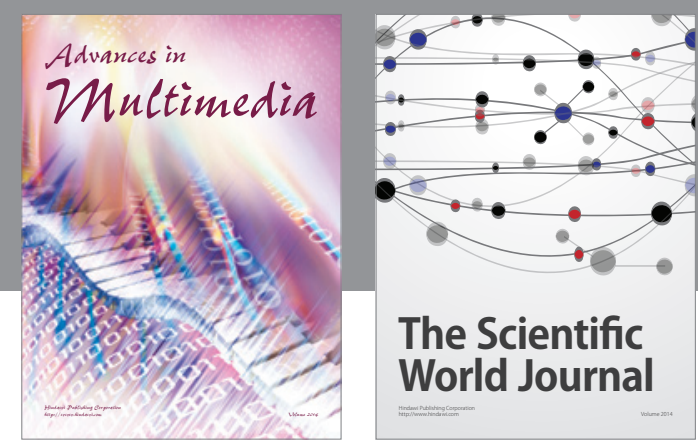

The Scientific World Journal
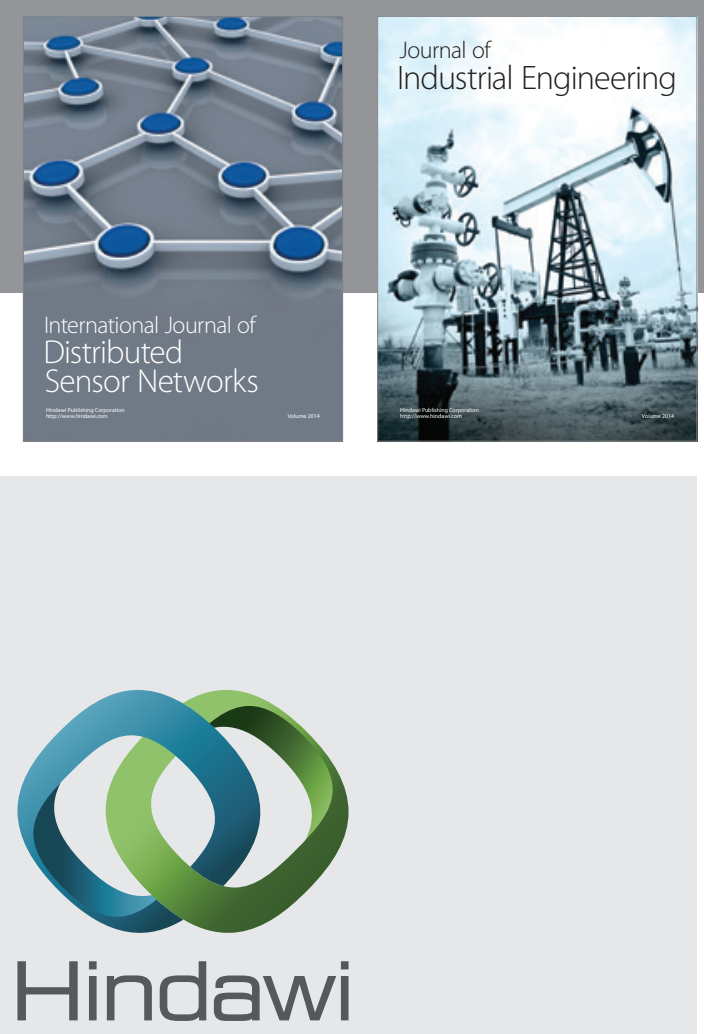

Submit your manuscripts at

http://www.hindawi.com

\section{Computer Networks} and Communications
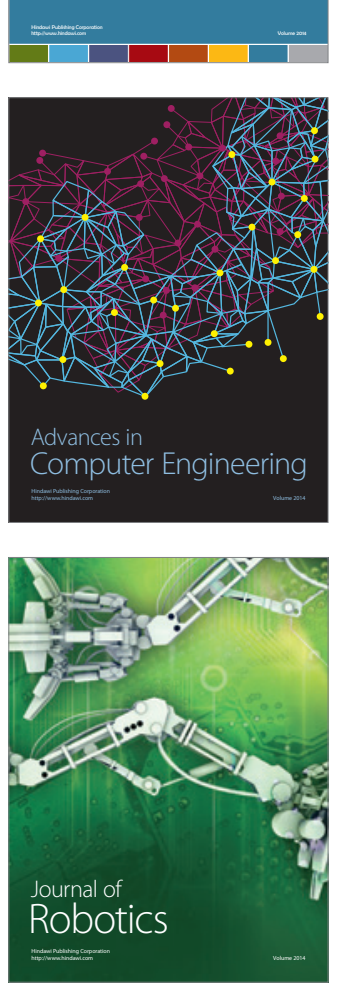
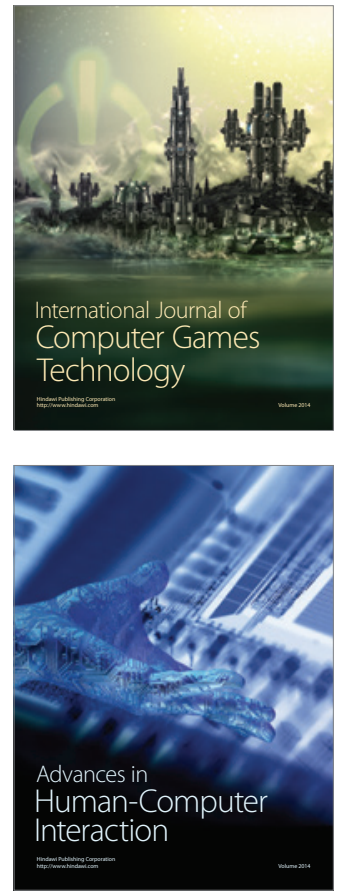
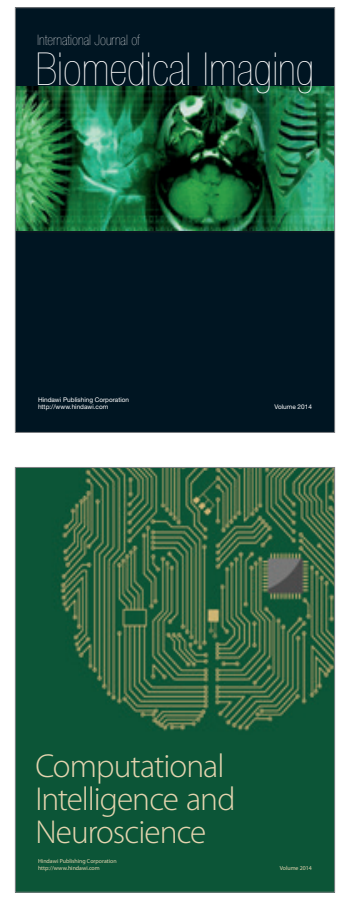
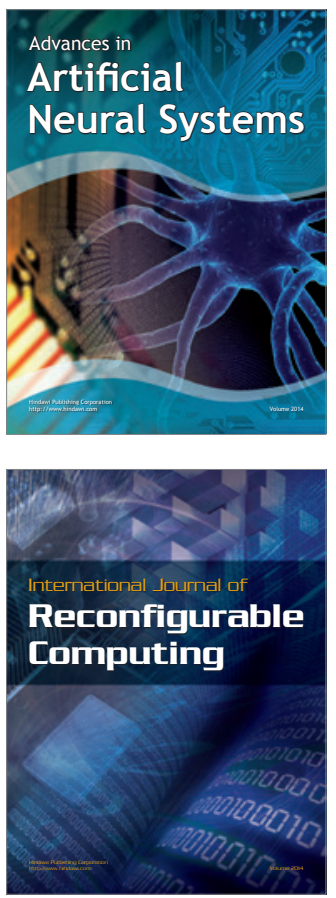
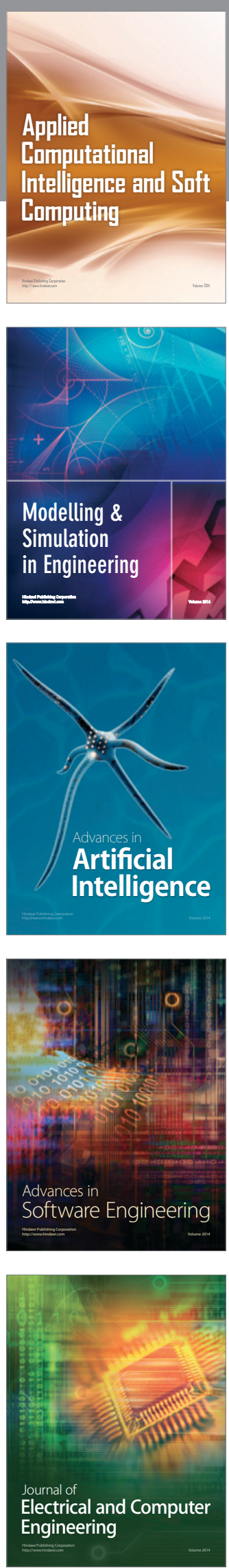